PROCEEDINGS OF THE

AMERICAN MATHEMATICAL SOCIETY

Volume 127, Number 9, Pages 2793-2795

S 0002-9939(99)04846-7

Article electronically published on April 15, 1999

\title{
ON THE DIMENSION OF ALMOST $n$-DIMENSIONAL SPACES
}

\author{
M. LEVIN AND E. D. TYMCHATYN \\ (Communicated by Alan Dow)
}

\begin{abstract}
Oversteegen and Tymchatyn proved that homeomorphism groups of positive dimensional Menger compacta are 1-dimensional by proving that almost 0-dimensional spaces are at most 1-dimensional. These homeomorphism groups are almost 0-dimensional and at least 1-dimensional by classical results of Brechner and Bestvina. In this note we prove that almost $n$-dimensional spaces for $n \geq 1$ are $n$-dimensional. As a corollary we answer in the affirmative an old question of R. Duda by proving that every hereditarily locally connected, non-degenerate, separable, metric space is 1-dimensional.
\end{abstract}

\section{INTRODUCTION}

We consider only separable metric spaces. A space $X$ is said to be almost $n$ dimensional if it has a basis $\left\{U_{i}\right\}$ such that if $\operatorname{cl} U_{i} \cap \operatorname{cl} U_{j}=\emptyset$, then $X=G \cup H$ where $G$ and $H$ are closed sets, $U_{i} \subset G \backslash H, U_{j} \subset H \backslash G$ and $\operatorname{dim} G \cap H \leq n-1$ and $n$ is the smallest natural number such that such a basis exists for $n$. It is clear that $n$-dimensional spaces are at most almost $n$-dimensional. We shall prove that for $n \geq 1$ the converse is also true. We shall also prove that if $X=X_{1} \cup X_{2}$ where $X_{1}$ is almost 0 -dimensional and $X_{2}$ is 0 -dimensional, then $\operatorname{dim} X \leq 1$.

A property equivalent to almost 0 -dimensionality was first considered in [7]. The Erdös space of irrational sequences in Hilbert space is known to be a universal almost 0-dimensional space [5]. Erdös space is 1-dimensional.

In [7] Erdös space was used to construct a hereditarily locally connected space (i.e., a connected space all of whose connected subsets are locally connected) which is not rim-countable. In [8] it was proved that hereditarily locally connected spaces are at most 2-dimensional. This was a partial solution to a question of R. Duda. In this paper we answer Duda's question in the affirmative by proving that hereditarily locally connected spaces are at most 1-dimensional.

A subset $X$ of a compactum $K$ is $L$-embedded in $K$ if for every open cover $\mathcal{U}$ of $K$ there is a neighbourhood $U$ of $X$ in $K$ such that the continua in $U$ refine $\mathcal{U}$. An almost 0 -dimensional space is $L$-embeddable in a compactum [6] and

Theorem 1.1 (Levin-Pol, [6]). If a space $X$ is L-embeddable in a compactum $K$, then $\operatorname{dim} X \leq 1$.

Received by the editors February 13, 1997 and, in revised form, November 20, 1997.

1991 Mathematics Subject Classification. Primary 54F45, 54F25, 54F50.

Key words and phrases. Almost 0-dimensional spaces, L-embeddings, hereditarily locally connected spaces.

The authors were supported in part by NSERC grant OGP0005616.

(C)1999 American Mathematical Society 


\section{Almost $n$-DIMENSIONAL SPACES}

Almost 0-dimensional spaces are at most 1-dimensional and the 1-dimensionality cannot be improved. Our first result shows that this interesting behavior does not occur in higher dimensions.

Theorem 2.1. If $X$ is almost $n$-dimensional, $n \geq 1$, then $X$ is $n$-dimensional.

Proof. Let $\mathcal{U}=\left\{U_{i}\right\}$ be a basis of open sets for $X$ which witnesses the almost $n$ dimensionality of $X$, i.e. if $\operatorname{cl} U_{i} \cap \operatorname{cl} U_{j}=\emptyset$ and $i<j$, then $X=G_{i j} \cup H_{i j}$ where $G_{i j}$ and $H_{i j}$ are closed sets, $U_{i} \subset G_{i j} \backslash H_{i j}, U_{j} \subset H_{i j} \backslash G_{i j}$ and $\operatorname{dim} G_{i j} \cap H_{i j} \leq n-1$. Let $X^{\prime}$ be a metric compactification of $X$. Index $\left\{\left(U_{i}, U_{j}\right): i<j\right.$ and $\left.\operatorname{cl} U_{i} \cap \operatorname{cl} U_{j}=\emptyset\right\}$ by $\left\{A_{k}\right\}_{k=1}^{\infty}$. If $A_{k}=\left(U_{i}, U_{j}\right)$, let $B_{k}=\mathrm{cl}_{X^{\prime}} G_{i j}$ and $C_{k}=\mathrm{cl}_{X^{\prime}} H_{i j}$. Form an inverse sequence as follows:

$X_{0}=X^{\prime}$

$X_{1}=B_{1} \times\{0\} \cup C_{1} \times\{1\} \subset X_{0} \times 2$ and let $\pi_{0}^{1}: X_{1} \longrightarrow X_{0}$ be the natural projection.

If spaces $X_{j}, j=0, \ldots, n$, and maps $\pi_{j}^{i}: X_{i} \longrightarrow X_{j}$ are defined for $j \leq i \leq n$, let $X_{n+1}=\left(\left(\pi_{0}^{n}\right)^{-1}\left(B_{n}\right) \times\{0\}\right) \cup\left(\left(\pi_{0}^{n}\right)^{-1}\left(C_{n}\right) \times\{1\}\right) \subset X_{n} \times 2 \subset X_{0} \times 2^{n+1}$. Let $\pi_{n}^{n+1}:$ $X_{n+1} \longrightarrow X_{n}$ be the natural projection and for $0 \leq j<n$ let $\pi_{j}^{n+1}: X_{n+1} \longrightarrow X_{j}$ be the $\operatorname{map} \pi_{j}^{n} \circ \pi_{n}^{n+1}$.

Let $\hat{X}^{\prime}=\lim _{\leftarrow}\left(X_{n}, \pi_{j}^{n}\right) \subset X^{\prime} \times 2^{\omega} . \hat{X}^{\prime}$ is a compactum. Let $\pi_{i}: \hat{X}^{\prime} \longrightarrow X_{i}$ be the projection. Then $\pi_{0}: \hat{X}^{\prime} \longrightarrow X_{0}=X^{\prime}$ is 0 -dimensional and onto. Let $\psi: \hat{X}^{\prime} \longrightarrow 2^{\omega}$ be the natural projection.

Let $\hat{X}=\pi_{0}^{-1}(X) \subset \hat{X}^{\prime} \subset X^{\prime} \times 2^{\omega}$. We show $\hat{X}$ is $L$-embedded in $\hat{X}^{\prime}$.

For each positive integer $n$ let $G_{n}=\bigcup\left\{U_{j}^{\prime}=X^{\prime} \backslash \operatorname{cl}_{X^{\prime}}\left(X \backslash U_{j}\right): U_{j} \in \mathcal{U}\right.$ and $\left.\operatorname{diam} U_{j} \leq 1 / n\right\}$ where $\operatorname{diam} U_{j}$ is determined with respect to a metric in $X^{\prime}$.

Then $G_{n}$ is open in $X^{\prime}$ and $X \subset G_{n}$. Let $C$ be a continuum in $\pi_{0}^{-1}\left(G_{n}\right)$. Then $\psi(C)$ is a singleton and, hence, $\operatorname{diam} C=\operatorname{diam} \pi_{0}(C)$ for the product metric in $X^{\prime} \times 2^{\omega}$. If $\operatorname{diam} \pi_{0}(C)>3 / n$, then there exist $U_{i}, U_{j} \in \mathcal{U}$ with $\operatorname{diam} U_{i}$, $\operatorname{diam} U_{j}<1 / n, i<j, \pi_{0}(C) \cap U_{i}^{\prime} \neq \emptyset, \pi_{0}(C) \cap U_{j}^{\prime} \neq \emptyset$ and $\operatorname{cl} U_{i} \cap \operatorname{cl} U_{j}=\emptyset$. Set $A_{k}=\left(U_{i}, U_{j}\right)$. Then $\pi_{k}(C)$ meets $X_{k-1} \times\{0\}$ and $X_{k-1} \times\{1\}$ since

$\pi_{k}\left(\pi_{0}^{-1}\left(U_{i}^{\prime}\right)\right) \subset \pi_{k}\left(\pi_{0}^{-1}\left(B_{k} \backslash C_{k}\right)\right) \subset X_{k-1} \times\{0\}$ and

$\pi_{k}\left(\pi_{0}^{-1}\left(U_{j}^{\prime}\right)\right) \subset \pi_{k}\left(\pi_{0}^{-1}\left(C_{k} \backslash B_{k}\right)\right) \subset X_{k-1} \times\{1\}$.

This is a contradiction as $\pi_{k}(C)$ is connected. Hence each continuum in $\pi_{0}^{-1}\left(G_{n}\right)$ has diam $\leq 3 / n$ and by Theorem $1.1 \hat{X}$ is at most 1 -dimensional.

Let $K=\bigcup_{k}\left(B_{k} \cap C_{k} \cap X\right)$. Clearly $\operatorname{dim} K \leq n-1$. It is easy to see that for every $x \in X \backslash K, \pi_{0}^{-1}(x)$ is a singleton. Note that $\left.\pi_{0}\right|_{\hat{X}}: \hat{X} \longrightarrow X$ is closed, 0dimensional and onto. Hence by Vainstein's second theorem ([3], p. 245, Theorem 4.3.10) $\operatorname{dim} X \leq n$. Clearly $\operatorname{dim} X \geq n$ and we have $\operatorname{dim} X=n$.

Corollary 2.2. If $X$ is a hereditarily locally connected, non-degenerate space, then $\operatorname{dim} X=1$.

Proof. By [7], Theorem 7.4.1, each pair of disjoint, closed, connected subsets of $X$ can be separated by a closed countable subset of $X$. Hence each basis for $X$ of open connected sets witnesses the almost 1-dimensionality of $X$. By Theorem 2.1 $X$ is 1-dimensional.

Theorem 2.3. Let $X=X_{1} \cup X_{2}$ where $X_{1}$ is almost 0-dimensional and $X_{2}$ is 0-dimensional. Then $\operatorname{dim} X \leq 1$. 
Proof. We may assume that $X_{1}$ is dense in $X$. Let $\mathcal{U}=\left\{U_{i}\right\}$ be a collection of open sets in $X$ such that $\left\{U_{i} \cap X_{1}\right\}$ is a basis of $X_{1}$ which witnesses the almost 0 -dimensionality of $X_{1}$. Since $X_{2}$ is 0 -dimensional each pair $\left(U_{i}, U_{j}\right)$ of $\mathcal{U}$ with $\operatorname{cl} U_{i} \cap \operatorname{cl} U_{j}=\emptyset$ can be separated by a 0 -dimensional closed subset. We use the same notation and construction as in the proof of Theorem 2.1. The difference between our case and the proof of Theorem 2.1 is that $\mathcal{U}$ is not a basis of $X$. Therefore we need a more subtle approach to show that $\hat{X}$ is $L$-embedded in $\hat{X}^{\prime}$.

$G_{n}$ covers $X_{1}$. Take a cover $\mathcal{V}_{n}$ of $X_{2}$ by open disjoint subsets of $X^{\prime}$ with $\operatorname{diam}<1 / n$ and let $V_{n}=\bigcup\left\{V: V \in \mathcal{V}_{n}\right\}$. Let $C$ be a continuum in $\pi_{0}^{-1}\left(G_{n} \cup V_{n}\right)$. If $\pi_{0}(C) \cap G_{n}=\emptyset$, then $\pi_{0}(C)$ is a subset of $V_{n}$ and clearly diam $\pi_{0}(C)<1 / n$.

If $\pi_{0}(C) \cap G_{n} \neq \emptyset$, then by the reasoning of the proof of Theorem 2.1 we get that $\operatorname{diam} \pi_{0}(C) \cap G_{n} \leq 3 / n$. As $V_{n}$ is the union of disjoint open sets $\pi_{0}(C) \subset O=$ $\left(\bigcup\left\{V: V \in \mathcal{V}_{n}, V \cap \pi_{0}(C) \cap G_{n} \neq \emptyset\right\}\right) \cup\left(\pi_{0}(C) \cap G_{n}\right)$. Clearly $\operatorname{diam} O<3 / n+2 / n$. Thus, $\hat{X}$ is $L$-embedded in $\hat{X}^{\prime}$.

Remark. Note that the union of two almost 0-dimensional spaces fails to be of $\operatorname{dim} \leq 1$. Indeed, let $Y$ be 1-dimensional and almost 0 -dimensional, let $M$ be a 1-dimensional compactum and let $M=M_{1} \cup M_{2}, \operatorname{dim} M_{1}=\operatorname{dim} M_{2}=0$. Then $X_{1}=Y \times M_{1}$ and $X_{2}=Y \times M_{2}$ are almost 0-dimensional, and by a theorem of Hurewicz [4] (see also [3], p. 78, 1.9.E(b)) $X=X_{1} \cup X_{2}=Y \times M$ is 2-dimensional.

\section{REFERENCES}

[1] Mladen Bestvina, Characterizing $k$-dimensional universal Menger compacta, Memoirs Amer. Math. Soc., 380(1988). MR 89g:54083

[2] Beverly Brechner, On the dimension of certain spaces of homeomorphisms, Trans. Amer. Math. Soc., 121(1966), 516-548. MR 32:4662

[3] R. Engelking, Theory of dimensions finite and infinite, Heldermann Verlag, Lemgo, 1995. MR 97j:54033

[4] W. Hurewicz, Sur la dimension des produits Cartesiens, Ann. of Math., 36(1935), 194-197.

[5] K. Kawamura, Lex G. Oversteegen and E. D. Tymchatyn, On homogeneous, totally disconnected, 1-dimensional spaces, Fund. Math., 150(1996), 97-112. MR 97d:54060

[6] Michael Levin and Roman Pol, A metric condition which implies dimension $\leq 1$, Proc. Amer. Math. Soc., 125(1997), no. 1, 269-273. MR 97e:54033

[7] T. Nishiura and E. D. Tymchatyn, Hereditarily locally connected spaces, Houston J. Math., 2(1976), 581-599. MR 55:9023

[8] Lex G. Oversteegen and E. D. Tymchatyn, On the dimension of certain totally disconnected spaces, Proc. Amer. Math. Soc., 122(1994), 885-891. MR 95b:54050

Department of Mathematics, Tulane University, New Orleans, Louisiana 70118-5698 E-mail address: mlevin@mozart.math.tulane.edu

Current address: Institute of Mathematics, Tsukuba University, Tsukuba, Ibaraki 305, Japan

E-mail address: mlevin@math.tsukuba.ac.jp

Department of Mathematics and Statistics, University of Saskatchewan, Saskatoon, CANADA S7N 0W0

E-mail address: tymchatyn@math.usask.ca 